\title{
Cancer Patients' Perspectives on Integrating Cancer Care in Primary Care Settings: A Qualitative Inquiry
}

\author{
Seit Mei Chien ${ }^{1,2}$, Fazean Idris ${ }^{2}$, Hashmet Parveen Ghouse ${ }^{2}$, Syafiq Abdullah', \\ Munikumar RamasamyVenkatasalu ${ }^{4}$
}

\begin{abstract}
${ }^{1}$ Department of Health Services, Ministry of Health, Commonwealth Drive, Bandar Seri Begawan BB3910, Brunei Darussalam. ${ }^{2}$ Institute of Health Sciences, Universiti Brunei Darussalam, Jalan Tungku Link, Gadong BE1410, Brunei Darussalam. ${ }^{3}$ Raja Isteri Pengiran Anak Saleha Hospital, Ministry of Health, Bandar Seri Begawan BA1710, Brunei Darussalam. ${ }^{4}$ Oxford School of Nursing and Midwifery, Faculty of Health and Life Sciences, Oxford Brookes University, MR1/02 Marston Road Campus, Jack Straws Lane, Headington Oxford OX3 0FL, United Kingdom.
\end{abstract}

\begin{abstract}
Introduction: Research has demonstrated the importance of general practitioners in providing care for cancer patients within the concept of 'care closer to home'. This study reports cancer patients' views and expectations on integrated cancer care in primary care settings in Brunei. Methods: A qualitative approach using semi-structured in-depth interviews with cancer patients were conducted. The interviews were recorded, transcribed, and analysed using thematic analysis. Results: 13 cancer patients participated and were interviewed, leading to three key themes that emerged from this study: (i) perceived challenges for providing cancer care at primary care settings; (ii) current health care system favours hospital-based cancer care and (iii) expectations towards integration of cancer care into primary care. Conclusion: Participants expressed their acceptance to the concept of primary care-based cancer care, mainly due to convenience and minimised waiting time. For this to be in place, there is a need for stronger communication channels between general practitioners and hospital physicians, familiarity of patients' cases among general practitioners, in-depth knowledge and experience of general practitioners in cancer care, and consulting the same general practitioners to provide continuity of care.
\end{abstract}

Keywords: Cancer care- patients' perspectives- integration- primary care- qualitative

\section{Introduction}

Globally, there is an increase in newly diagnosed cancer patients and cancer survivors as a result of growing and aging population, and technological advances in cancer care [1-2]. Conventional hospital based cancer care causes a significant burden on tertiary care, yet it is unclear whether it provides early diagnosis of recurrence and improve survival [3]. Meanwhile, primary care is increasingly promoted by governments worldwide as the preferred place for cancer care, due to health care costs and patients' preferences [4]. Evidence is emerging that there are no differences in cancer patients' overall well-being, recurrence rates, survival between primary and hospital-based follow up, with primary care found to be more cost-effective [5-6].
Integrated cancer care between primary and tertiary care settings is recently evidenced as a successful model of care. Indeed, integrated follow up in primary care for people with breast and colorectal cancers showed high patient satisfaction and no adverse outcomes [7-8]. A study assessing general practitioners' (GP) attitudes toward follow-up after cancer treatment showed that they felt confident in doing so and would like to contribute to long-term care of cancer patients [9]. Meanwhile, patients had also reported their views for greater involvement of GPs in all aspects of their cancer care [10] and cancer survivors had shown satisfaction with primary care delivery [11]. It has been predicted that integrated cancer care may save up to $75 \%$ of health care costs [12].

\section{Corresponding Author:}

Dr. Seit Mei Chien

Department of Health Services, Ministry of Health, Commonwealth Drive, Bandar Seri Begawan BB3910, Brunei Darussalam.

Email: meichien.seit@gmail.com 
In Brunei, cancer has been the leading cause of mortality from 2009 to 2017 , with $19.3 \%$ of deaths in 2017 [13]. At this stage, cancer patients' expectations on integrating cancer care into local primary care settings are not yet known. This study reports cancer patients' experiences, preferences, and expectations towards the integration of cancer care in primary care in Brunei.

\section{Materials and Methods}

\section{Design, Setting and Participants}

We adopted a qualitative approach and considered that semi-structured in-depth interviews with cancer patients were the most appropriate research technique to capture our research aims. Cancer patients aged between 18 and 80 years old attending the oncology outpatient clinic in the main hospital in Brunei undergoing either active treatment, palliative care, or in remission, were included in the study. Following ethical approval, nurses working in the oncology clinic approached eligible patients. Patients who agreed to participate were given a participant information sheet and further explanation about the study by the main researcher (SMC) and were interviewed at an agreed date, time and location.

\section{Data Collection}

Semi-structured in-depth interviews were conducted between February to November 2017 with thirteen participants. An interview guide (Table 1) derived from literature review and consensus of research team was developed with a view to obtain participants' experiences, views and expectations towards their cancer care in primary care. We operationally defined cancer care as care provided when patients were receiving diagnosis, referral, active treatment, palliative care and related follow-up care in cancer.

$\mathrm{SMC}$, the main researcher, was guided by a senior investigator (MRV), with vast experience in conducting and analysing interviews on cancer-sensitive topics. The interviews lasted between 22 to 60 minutes and were conducted in private rooms at various places - health centres, oncology clinic, the university where the researchers were based, participants' home and participants' workplace as per mutually agreed. As the questions asked may be sensitive to participants, necessary steps were taken to ensure that participants felt comfortable and were reassured about confidentiality and anonymity. All forms of communication with participants were conducted in a supportive way and appropriate empathic responses were used to acknowledge their distress. We followed the principles of qualitative research [14] and ended further interviews when data saturation was reached at the $13^{\text {th }}$ interview.

\section{Data Analysis}

The interviews were digitally recorded, transcribed verbatim, and analysed using thematic analysis [15]. Firstly, the transcripts were read to get an immersion into the interview data. Subsequently, initial codes were developed; the coded units which represented the different aspects of participants' experiences, preferences, and expectations towards integration of cancer care in primary care settings were identified. The coded data was compiled under wider subthemes, which were compared to the original transcripts for consistency and contextual verification. Consequently, the contents in each subtheme were summarised, which generalised the descriptions concerning the research topic. SMC and MRV coded the data together and agreed on the final themes. An audit trial was performed by SMC and MRV to enhance trustworthiness of the study.

\section{Ethical Considerations}

The study was approved by the Ministry of Health Research and Ethics Committee and Ethics committee of PAPRSB Institute of Health, Universiti Brunei Darussalam (UBD/IHS/B3/8).

\section{Results}

The general characteristics of the participants are shown in Table 2. There were ten females and three males of median age 50 years. The most prevalent cancers were lung and breast. Most were diagnosed with cancer within the last 5 years.

Data analysis led to three main themes of participants' range of perspectives on primary care-based cancer care: (i) perceived challenges for providing cancer care at primary care settings, (ii) current health care system favours hospital-based cancer care and (iii) expectations towards integration of cancer care into primary care, further explained below.

\section{Theme 1: Perceived challenges for providing cancer care} at primary care settings

Most participants regarded cancer as a specialised area requiring specialist knowledge, thus their care would be most appropriately followed up in a hospital setting than in primary care. Participant 6 questioned the ability of cancer care at the primary care, as described below:

'Hospital has specialist for cancer. For my case, I am categorised as severe, so if I go to the hospital, then I would be handled with the proper care because hospital is specific for cancer.' (P6)

A few participants felt that hospital physicians would do more thorough investigations instead of the GP, as questioned by Participant 10:

'Why does specialist do all sorts of investigations but the normal doctor in clinic does not?' (P10)

A few participants presented to the GP repeatedly with the same complaint. However, the symptoms of cancer were disregarded by the GPs and participants did not agree with the diagnosis, as illustrated by Participant 13:

'They (GPs) examined my abdomen. They just pressed like that. They just said it was gastric..... I mean they should check thoroughly. They should focus on where exactly the pain is. They (GPS) just gave me reflux medication. After two to three weeks, I would go to the health centre again. Same problem. It was always the same. They did nothing. '(P13) 
Table 1. Interview Guide

\begin{tabular}{ll}
\hline Interview guide & Questions \\
\hline Experiences & $\cdot$ How were you diagnosed with cancer? \\
& $\cdot$ When did you last see the GP regarding your concerns related to cancer? \\
& $\cdot$ Were there any problems in seeing the GP regarding your cancer? \\
Views & $\cdot$ Do you think the GP can detect your cancer early? \\
& $\cdot$ How do you think the GP can be involved in your care? \\
& $\cdot$ Do you think the GP can look after cancer patients? Are there challenges in this? \\
& $\cdot$ Would you like the GP to know if you are admitted in hospital? How could this information help? \\
& $\cdot$ How can hospital doctors and GPs work together to help cancer patients? \\
Expectations & $\cdot$ Are there any areas where you would like your case to be taken over by the GP rather than the hospital? \\
& $\cdot$ What kinds of support would be most useful/beneficial for you and other patients with cancer, which can be \\
& provided by primary care?
\end{tabular}

Participants also reported that the GPs lacked knowledge and skills of managing cancer. As a result, they felt that follow up in the hospital setting would be more ideal, as illustrated by Participant 11:

'When one goes to the area clinic, I am afraid the doctor does not know the story even though there is BruHIMS (unique patient electronic medical record number). One will not feel comfortable, needing to tell again what happened.' (P11)

On the other hand, we also found overall positive experiences from participants who were satisfied with the services provided in the primary care. Participant 7 stated that she was referred promptly to the hospital for further evaluation of the possibility of cancer:

'At first I had fever. So, I went to ' $X$ ' Health Centre. The doctor requested for blood tests. From there, it was found and I was sent straight to the hospital for further evaluation.' (P7)

Some participants agreed that utilising primary care would reduce patient load at acute hospital. Participant 7 reported that cancer care at primary care settings would provide more choice of doctors than a specialist doctor at acute hospital, hence, cancer care should be 'delegated.'

'Don't rely only on one doctor - I sympathise. The patients are a lot (in hospital). So, if possible, the tasks can be delegated.' (P7)

Most of the participants reported the benefits of a primary care-based cancer care would include care closer to home, convenience, availability of doctors, and less waiting time.

'The doctors are always there (in health centres). Waiting time is not too long. It is good. I just register and pay a dollar and waiting time to see the doctor is not long.' (P12).

Theme 2: Current health care system favours hospitalbased cancer care

Participants reported that current cancer care pathway mainly favours hospitals than primary care. Currently in Brunei, patients who were referred from primary care for further evaluation of possibility of cancer diagnosis would continue to be cared for by the hospital physician from diagnosis, treatment, and surveillance phases. This lead to cancer patients approaching the hospital physicians only for any cancer-related issues instead of their GPs who were not engaged in any aspects of their cancer care, as mentioned by Participant 2:

'Since I was diagnosed with cancer, I have only been going to the main hospital. I have not been to the health centre.' (P2)

Some preferred a hospital-based cancer care pathway as they felt more comfortable talking to the same physicians whom they were already familiar with since their cancer diagnosis.

'Mostly patients feel comfortable with the doctors in the main hospital. They will prefer to go to hospital only.' (P11)

Participants also reported other system errors such as the availability of cancer medications only in tertiary hospital, and inflexible and rigid working hours of primary care confined to the normal working hours. These system factors favoured more towards hospital settings to provide cancer care, as illustrated by Participants 2 and 9:

'Well, I just go straight to hospital because they have emergency in hospital. And the health centre here, it is only opened during office hours. '(P2)

'There is an issue if I collect my medication here (health centre), as my cancer medication is not available here. I will need to go to hospital. '(P9)

Theme 3: Expectations towards integration of cancer care into primary care

It was clear in most participants' discussions that they were open to the concept of primary care-based cancer care, and that cancer care should not be based only in the hospital setting. Indeed, participants suggested possible ways for integration between primary and secondary care. For example, participant 4 commented on 'oncologist coming to the health centre'. Apart from that, participant 12 felt that 'there should be constant communication between GPs and hospital physicians'.

Participant 10 reported that she would prefer to see the hospital physician occasionally still while GPs could do the more regular routine follow up. In order for this to take place, both doctors from the primary and secondary care should be working together. 
Table 2. General Characteristics of Study Participants

\begin{tabular}{lccccccc}
\hline Participant & Sex & Age & Profession & Education & Year of cancer diagnosis & Location of cancer & Treatment \\
\hline P1 & F & 35 & Housewife & High school & 2009 & Brain & Surgery, radiotherapy \\
P2 & M & 40 & Police & High school & 2014 & Colorectal & Surgery, radiotherapy, chemotherapy \\
P3 & M & 62 & Retired & University & 2009 & Lung & Radiotherapy, chemotherapy \\
P4 & F & 67 & Retired & High school & 2016 & Lung & Chemotherapy \\
P5 & F & 63 & Housewife & High school & 2008 & Lymphoma & Chemotherapy \\
P6 & F & 47 & Self-employed & Primary school & 2009 & Breast & Surgery, hormonal therapy \\
P7 & F & 50 & Teacher & University & 2013 & Rectal & Surgery, chemotherapy \\
P8 & F & 55 & Housewife & High school & 2016 & Lung & Patient refused treatment \\
P9 & F & 68 & Housewife & High school & 2016 & Breast & Hormonal therapy \\
P10 & F & 51 & Operator & High school & 2000,2014 & Thyroid, liver & Surgery, radiotherapy, chemotherapy \\
P11 & F & 48 & Dental nurse & University & 2014 & Breast & Surgery, chemotherapy \\
P12 & M & 26 & Officer & High school & 2016 & Brain & Radiotherapy, chemotherapy \\
P13 & F & 42 & Cleaner & High school & 2014 & Liver & Surgery \\
\hline
\end{tabular}

'It is ok (for cancer care in the community). Like I said, as long as there is a connection with $\mathrm{Dr}$ ' $X$ ' (hospital physician). Once in a while, patient can still see Dr ' $X$ '. Not every 1-3 months, but once in a while. Still it is important to see him. He is like the director of the movie. But for the routine appointments, we can see the GPs.'(P10)

As of now, patients in primary care settings may not necessarily see the same GPs, which may lead to unfamiliarity of cases and needing the patients to explain what had happened, as commented by Participant 10:

'Sometimes doctors (GPS) do not check the files thoroughly. So, I have to explain everything from A to $Z$ again. So, it is better for me to go to my own doctor (in hospital).'(P10)

In order to overcome this issue, there has been a suggestion of 'seeing the same doctor' in primary care so to avoid confusion as different doctors would have different approaches to management plans, as illustrated by Participant 13:

'When we see the doctor, he will say this. For the review appointment, a different doctor will say different things. So which advice do we follow? We don't know. We are just following doctor's advice. If possible, it would be better with the same doctor.' (P13)

\section{Discussion}

Many existing studies on primary-based cancer care have reported cancer patients' attitudes, health behaviours, preferences and perspectives [16-19], whereas our study focused on cancer patients' experiences and expectations towards integrated cancer care in primary care.

Firstly, inconsistent assessment and diagnostic procedures among GPs seems to frustrate cancer patients, hence leading to multiple visits to primary care. The National Cancer Diagnosis Audit in the United Kingdom showed that $26 \%$ of patients had three or more GP consultations before being referred to hospital care for further evaluation [20]. Lyratzopoulos et al. [21] showed that some patients experienced multiple consultations leading to prolong intervals to specialist referral and assessment for suspected cancer. Mendonca et al. [22] also reported $40 \%$ of patients who had multiple GP consultations were not satisfied with how hospital physicians and GPs collaborate. Cancer diagnosis remains challenging in primary care as cancer patients present to GPs without any cancer alarming symptoms as shown in a study by Jensen et al. [23]. In the United Kingdom, cancer care two-week wait referral pathways aim to improve patients' satisfaction, reduce waiting times to be seen by specialists, and earlier diagnosis, which would result in better prognosis of patients [24]. Yet, there is no evidence available on development and test of integrated cancer care pathway for assessment, diagnosis and referral of suspected cancer.

Misperceptions about primary care-based cancer care as an avenue for 'minor illness' management among cancer patients often limit integration of cancer care in primary care settings [25]. Our study participants believed that their cancer care would require a specialists' expertise of cancer, of which GPs do not acquire. Hence, they reported their refusal to visit primary care as the GPs would still refer them to hospital eventually, similar to a Danish study [26]. Patients expressed strong preferences for quick diagnostic evaluation after initial presentation to GPs and would choose to undergo investigations for suspected cancer even if their risk was as low as $1 \%$ [27]. In contrast, GPs in other studies reported that they valued their role as gatekeeper and perceived their skills as being able to identify patients who needed further work up and referral to hospital from those who were able to manage in primary care [28]. Public awareness interventions on role of GPs in cancer care may challenge such misperceptions.

In view of the increasing cancer survival rates, managing cancer patients in the primary care has been identified as a key element for future-effective and cost-effective cancer care [29]. Participants reported that cancer care tasks from hospital could be delegated to primary care. Indeed, the roles of primary care-based cancer care are also widely accepted by cancer survivors, similar to many studies [5]. Participants also reported care closer to home, and easy accessibility of GPs, as advantages of integrated cancer care in primary care [4- 
30]. Yet, lack of cancer diagnostic skills, limited education, knowledge and skills, experience, and available time of GPs were perceived barriers by patients for primarybased cancer care [26]. Mao et al. reported that half of the patients with breast cancers had concerns about GPs' ability to address patients' cancer-specific issues [31]. In another qualitative study, cancer patients did not see their GPs, because they felt that the GPs were too busy or to be lacking in oncology knowledge [32].

In the current tertiary-based cancer care centre in Brunei, similar to many countries, cancer patients seem to lose their follow up by GPs [33]. Another possible explanation may be because in developing countries, cancer patients are still traditionally followed up in hospital setting, as compared to developed countries, whereby there is already a gradual shift of cancer care from secondary to primary care settings [34]. Patients in our studies, similar to other studies, prefer doctors whom they are most familiar with, and who oversaw them during their active cancer treatment [35]. This could be due to the strong relationship built between the patients and hospital physicians during patients' active treatment [31-32]. Therefore, the current structure of cancer care has led our participants to approach the hospital for their cancer care instead of attending primary care.

In contrast, other studies reported that patients who were already receiving cancer follow-up from their GPs were satisfied with the care provided and did not report any drawbacks [25-31]. For example, Nyarko et al. [11] reported high satisfaction rates on primary care delivery among cancer survivors. Hence, integrated pathways should empower earlier involvement of GPs during the active cancer treatment stage that may increase patients' confidence in primary care-based follow-up. The participants in our study also discussed about the inflexibility of opening hours of primary care. Similarly, many patients also felt that it was difficult to approach GPs after office hours [36]. In fact, a study by Borgsteede et al. [37] reported the main factor for effective out of hours cancer care is the accessibility of GPs and nursing support.

Our study provided evidence for participants, acceptance towards integrated cancer care at primary care settings, conditional in having clear roles and responsibilities of GPs and hospital physicians, effective communication between GPs and hospital physicians, guidance on follow-up protocols and common treatments, knowledgeable GPs in cancer care and rapid access to specialists [26]. Indeed, such integrated care with GPs and hospital physicians show no adverse outcomes in patients with bowel and breast cancers and can provide high patient satisfaction rates [7-8]. On the other hand, our study also showed that GPs were unfamiliar with their cases leading to poor satisfaction and frustration among cancer patients in primary care. This is similar to studies by Thind et al. [25] and Roorda et al. [26], which reported lack of GPs' knowledge on patients' histories was regarded as a disadvantage.

To overcome such inconsistent approach, patients valued and preferred to be followed up by the same care provider at each visit because of the established doctor-patient relationship as well as physicians' knowledge and familiarity on the patients' histories [33-35]. Seeing the same GPs would also cause less confusion in management plans and also remain as effective way of securing good information, thus enable GPs to provide seamless care along the entire cancer care spectrum [38]. Continuity of cancer care would result in better communication, stronger relationship between GPs and patients, allow patients to cope better, enhance patient access to care, and improve overall experiences for cancer patients [39].

This study was not without limitations. Firstly, as many had their cancer diagnosis more than 5 years ago, there may be recall bias as information provided was relied on what participants reported and might had affected the accuracy of recalling the actual experiences. Secondly, the interviewer was a GP who may influence the participants' views, despite our reassurance.

In conclusion, this study adds to the growing existing evidence base looking at factors and barriers of integrating cancer care in primary care. We found that our cancer patients were receptive to the idea for cancer care to be integrated in primary care. However, in order for this to take place, it is important to ensure established communication channels between GPs and hospital physicians, deeper understanding of cancer cases among GPs, improved cancer care knowledge and experience of GPs, and consulting the same GPs to provide continuity of care, as factors that enable quality cancer care at primary care settings in Brunei. Thus, policy makers should incorporate these elements of integration in the implementation of cancer care into primary care, especially for Brunei before we are ready to integrate a primary care cancer care. Furthermore, future research needs to address GPs' knowledge gaps in cancer care and to explore development and test of integrated cancer care pathways in primary care settings.

\section{Acknowledgements}

The authors would like to express their sincere gratitude to the nurses working in the outpatient clinics of the Oncology Department of Raja Isteri Pengiran Anak Saleha Hospital who assisted in the recruitment process of patients.

This research did not receive any specific grant from funding agencies in the public, commercial, or not-forprofit sectors. The authors declare no conflict of interest.

\section{References}

1. American Society of Clinical Oncology. The state of cancer care in America, 2017: A report by the American Society of Clinical Oncology. J Oncol Pract, 2017;13(4):e353-e94. doi: 10.1200/JOP.2016.020743

2. Miller KD, Siegel RL, Lin CC, Mariotto AB, Kramer JL, Rowland $\mathrm{JH}$, et al. Cancer treatment and survivorship statistics, 2016. CA Cancer J Clin, 2016;66(4):271-89. doi: 10.3322/caac. 21349

3. Francken AB, Bastiaannet E, Hoekstra HJ. Follow-up in patients with localised primary cutaneous melanoma. 
Lancet Oncol, 2005;6(8):608-21. DOI: 10.1016/S14702045(05)70283-7

4. Rubin G, Berendsen A, Crawford SM, Dommett R, Earle C, Emery J, et al. The expanding role of primary care in cancer control. Lancet Oncol, 2015;16(12):1231-72. doi: 10.1016/ S1470-2045(15)00205-3

5. Emery JD, Jefford M, King M, Hayne D, Martin A, Doorey J, et al. ProCare Trial: a phase II randomized controlled trial of shared care for follow-up of men with prostate cancer. BJU Int, 2017;119(3):381-9. doi: 10.1111/bju.13593

6. Emery JD, Shaw K, Williams B, Mazza D, Fallon-Ferguson J, Varlow M, et al. The role of primary care in early detection and follow-up of cancer. Nat Rev Clin Oncol, 2013;11(1). doi: 10.1038/nrclinonc.2013.212

7. Grunfeld E, Levine MN, Julian JA, Coyle D, Szechtman B, Mirsky D, et al. Randomized trial of long-term follow-up for early-stage breast cancer: a comparison of family physician versus specialist care. J Clin Oncol, 2006;24(6):848-55. DOI: $10.1200 /$ JCO.2005.03.2235

8. Wattchow DA, Weller DP, Esterman A, Pilotto L, McGorm K, Hammett Z, et al. General practice vs surgical-based followup for patients with colon cancer: randomised controlled trial. Br J Cancer, 2006;94(8):1116-1121. doi: 10.1038/ sj.bjc. 6603052

9. Fidjeland HL, Brekke M, Vistad I. General practitioners' attitudes toward follow-up after cancer treatment: A crosssectional questionnaire study. Scand J Prim Health Care, 2015;33(4):223-32. doi: 10.3109/02813432.2015.1118836

10. Aubin M, Vézina L, Verreault R, Fillion L, Hudon É, Lehmann F, et al. Patient, primary care physician and specialist expectations of primary care physician involvement in cancer care. J Gen Intern Med, 2012;27(1):815. doi: $10.1007 / \mathrm{s} 11606-011-1777-7$

11. Nyarko E, Metz JM, Nguyen GT, Hampshire MK, Jacobs LA, Mao JJ. Cancer survivors' perspectives on delivery of survivorship care by primary care physicians: an internetbased survey. BMC Fam Pract, 2015;16(1):143. doi: 10.1186/s12875-015-0367-x

12. Gilbert S, Reid KR, Lam MY, Petsikas D. Who should follow up lung cancer patients after operation? Annals of Thoracic Surgery, 2000;69(6):1696-700.

13. Ministry of Health Brunei Darussalam (2018). Department of Policy and Planning. Health Information Booklet 2017. Retrieved from http://www.moh.gov.bn/SitePages/Health Information Booklet.aspx

14. Saunders B, Sim J, Kingstone T, Baker S, Waterfield J, Bartlam B, et al. Saturation in qualitative research: exploring its conceptualization and operationalization. Qual Quant, 2018;52: 1893. https://doi.org/10.1007/s11135-017-0574-8

15. Nowell LS, Norris JM, White DE, Moules NJ. Thematic Analysis: Striving to meet the trustworthiness criteria. International Journal of Qualitative Methods, 2017. https:// doi.org/10.1177/1609406917733847

16. Aubin M, Vézina L, Verreault R, Fillion L, Hudon É, Lehmann F, et al. Family physician involvement in cancer care follow-up: the experience of a cohort of patients with lung cancer. Ann Fam Med, 2010;8(6):526-32. doi: 10.1370/ afm. 1171

17. Arora NK, Reeve BB, Hays RD, Clauser SB, Oakley-Girvan I. Assessment of quality of cancer-related follow-up care from the cancer survivor's perspective. J Clin Oncology, 2011;29(10):1280.doi: 10.1200/JCO.2010.32.1554

18. Snyder CF, Frick KD, Herbert RJ, Blackford AL, Neville BA, Carducci MA, et al. Preventive care in prostate cancer patients: following diagnosis and for five-year survivors. J Cancer Survivor, 2011;5(3):283-91. doi: 10.1007/s11764-

\section{1-0181-y}

19. previously 20 Hewitt ME, Bamundo A, Day R, Harvey C. Perspectives on post-treatment cancer care: qualitative research with survivors, nurses, and physicians. J Clin Oncology, 2007;25(16):2270-3. DOI:10.1200/ JCO.2006.10.0826

20. Swann R, McPhail S, Witt J, Shand B, Abel GA, Hiom S, et al. Diagnosing cancer in primary care: results from the National Cancer Diagnosis Audit. Br J Gen Pract, 2018; 68(666):e63-e72. doi: 10.3399/bjgp17X694169

21. Lyratzopoulos G, Neal RD, Barbiere JM, Rubin GP, Abel GA. Variation in number of general practitioner consultations before hospital referral for cancer: findings from the 2010 National Cancer Patient Experience Survey in England. Lancet Oncol, 2012;13(4):353-65. doi: 10.1016/S14702045(12)70041-4.

22. Mendonca SC, Abel GA, Saunders CL, Wardle J, Lyratzopoulos G. Pre-referral general practitioner consultations and subsequent experience of cancer care: evidence from the English Cancer Patient Experience Survey. Eur J Cancer Care, 2016;25(3):478-90. doi: 10.1111/ ecc. 12353

23. Jensen H, Tørring ML, Olesen F, Overgaard J, Vedsted P. Cancer suspicion in general practice, urgent referral and time to diagnosis: a population-based GP survey and registry study. BMC Cancer, 2014;14(1):636. doi: 10.1186/14712407-14-636.

24. Prades J, Espinas J, Font R, Argimon J, Borras J. Implementing a cancer fast-track programme between primary and specialised care in Catalonia (Spain): a mixed methods study. Br J Cancer, 2011;105(6):753. doi: 10.1038/ bjc. 2011.308

25. Thind A, Liu Y, Maly RC. Patient satisfaction with breast cancer follow-up care provided by family physicians. J Am Board Fam Med, 2011;24(6):710-6. doi: 10.3122/ jabfm.2011.06.100288

26. Roorda C, Bock GH, Scholing C, Meer K, Berger MY, Fouw M, et al. Patients' preferences for post-treatment breast cancer follow-up in primary care vs. secondary care: a qualitative study. Health Expect, 2015;18(6):2192-201. doi: 10.1111/hex.12189

27. Banks J, Hollinghurst S, Bigwood L, Peters TJ, Walter FM, Hamilton W. Preferences for cancer investigation: a vignettebased study of primary-care attendees. Lancet Oncol, 2014;15(2):232-40. doi: 10.1016/S1470-2045(13)70588-6

28. Green T, Atkin K, Macleod U. Cancer detection in primary care: insights from general practitioners. Br J Cancer, 2015;112(s1):S41. doi: 10.1038/bjc.2015.41

29. Emery J, Trevena L, Mazza D, Fallon-Ferguson J, Shaw K, Williams B, et al. The role of primary and community-based health care professionals in early detection and follow-up in cancer care - a rapid review of best practice models: an Evidence Check rapid review brokered by the Sax Institute (http://www.saxinstitute.org.au) for the Cancer Institute. NSW, 2012.

30. Mitchell GK. How well do general practitioners deliver palliative care? A systematic review. Palliat Med, 2002;16(6):457-64. DOI: 10.1191/0269216302pm573oa

31. Mao JJ, Bowman MA, Stricker CT, DeMichele A, Jacobs L, Chan D, et al. Delivery of survivorship care by primary care physicians: the perspective of breast cancer patients. J Clin Oncology, 2009;27(6):933-8. doi: 10.1200/ JCO.2008.18.0679

32. Pennery E, Mallet J. A preliminary study of patients' perceptions of routine follow-up after treatment for breast cancer. European Journal of Oncology Nursing, 
2000;4(3):138-45. DOI: https://doi.org/10.1054/ ejon.2000.0092

33. Anvik T, Holtedahl KA, Mikalsen H. "When patients have cancer, they stop seeing me"-the role of the general practitioner in early follow-up of patients with cancer-a qualitative study. BMC Fam Pract, 2006;7(1):19. DOI: 10.1186/1471-2296-7-19

34. Erikson C, Salsberg E, Forte G, Bruinooge S, Goldstein M. Future supply and demand for oncologists: challenges to assuring access to oncology services. J Oncol Pract, 2007;3(2):79-86. doi: 10.1200/JOP.0723601

35. Kimman ML, Dellaert BG, Boersma LJ, Lambin P, Dirksen CD. Follow-up after treatment for breast cancer: one strategy fits all? An investigation of patient preferences using a discrete choice experiment. Acta Oncol, 2010;49(3):328-37. doi: $10.3109 / 02841860903536002$

36. Foster J, Dale J, Jessopp L. A qualitative study of older people's views of out-of-hours services. Br J Gen Pract, 2001;51(470):719-23.

37. Borgsteede SD, Graafland-Riedstra C, Deliens L, Francke AL, van Eijk JT, Willems DL. Good end-of-life care according to patients and their GPs. Br J Gen Pract, 2006;56(522):20-6.

38. Haggerty JL, Roberge D, Freeman GK, Beaulieu C. Experienced continuity of care when patients see multiple clinicians: a qualitative metasummary. Ann Family Med, 2013;11(3):262-71. doi: 10.1370/afm.1499.

39. Easley J, Miedema B, Carroll JC, O’Brien MA, Manca DP, Grunfeld E. Patients' experiences with continuity of cancer care in Canada: results from the canimpact study. Can Fam Physician, 2016;62(10):821-7.

c) (i) (8)

This work is licensed under a Creative Commons AttributionNon Commercial 4.0 International License. 